\title{
INCORPORATING FAIRNESS IN GENERALIZED GAMES OF MATCHING PENNIES
}

\author{
RICHARD T. BOYLAN AND SIMON GRANT
}

\begin{abstract}
We examine individual behavior in generalized games of matching pennies. Our findings are twofold. First, individuals cooperate in these games; i.e., they systematically select strategies that lead both players to obtain higher expected payoffs than Nash. Second, among the main models in the extant literature (some of which were explicitly designed to induce cooperative behavior) the only one that predicts the observed behavior is the quantal response equilibrium.
\end{abstract}

\section{INTRODUCTION}

In this paper we examine experimental behavior in 'generalized games of matching pennies,' a class of two-by-two games with no pure strategy Nash equilibria described in Figure 2. Prior work has shown that the quantal response model fits the data better than Nash equilibrium or noisy Nash equilibrium [5], [7], [3], [4]. The existing papers have emphasized that the quantal response model is to be preferred because it models individuals as being boundedly rational. In this paper we discuss a different property of the quantal response model: it is the only existing model that leads to cooperative behavior and outcomes in the generalized game of matching pennies.

In section 2 we summarize the experimental findings for the generalized game of matching pennies. In section 3 we describe the quantal response equilibrium and show that its comparative statics are consistent with the experimental findings. We then discuss other models in section 4 and show how they all lead to predictions inconsistent with the experimental results. The Appendix contains all proofs.

Date: March 7, 2006.

We thank Jack Ochs for making his data available to us. Discussions with David Levine and Rick Wilson were also helpful. 


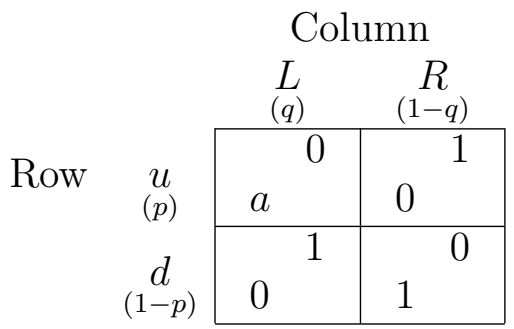

FiguRE 1. Generalized game of matching pennies, where $a>0$.

\section{STYLIZED FACTS}

In the unique Nash equilibrium of the asymmetric game of matching pennies, Row selects $u$ with probability $p=1 / 2$ and Column selects $L$ with probability $q=1 /(1+a)$. In particular, the parameter $a$ ('the own payoff') does not affect the equilibrium mixed strategy of Row. The experimental evidence summarized in Table 1 contradicts this prediction. Column $p^{A}$ denotes the frequency with which Row players selected action $u$. For each of the three sets of experiments (i.e., Goeree and Holt [3], Ochs [7] and McKelvey et al. [6]) $p^{A}$ is increasing in its own payoff $a$.

Table 1 provides further evidence against the predictions of the Nash equilibrium. Column $\pi_{R}$ is the expected payoff for Row given that the players select Nash equilibrium strategies. Column $\pi_{R}^{A}$ is the expected payoff for Row given the actual strategies selected by the players. It can be seen that when $a \neq 1$ players select strategies that lead to higher expected payoffs for the Row player than Nash. Similarly, Column player also obtains higher expected payoffs than Nash. For the experiment with $a=7$, Row obtains $27 \%$ more than the Nash expected payoffs while Column obtains $63 \%$ more than Nash. 


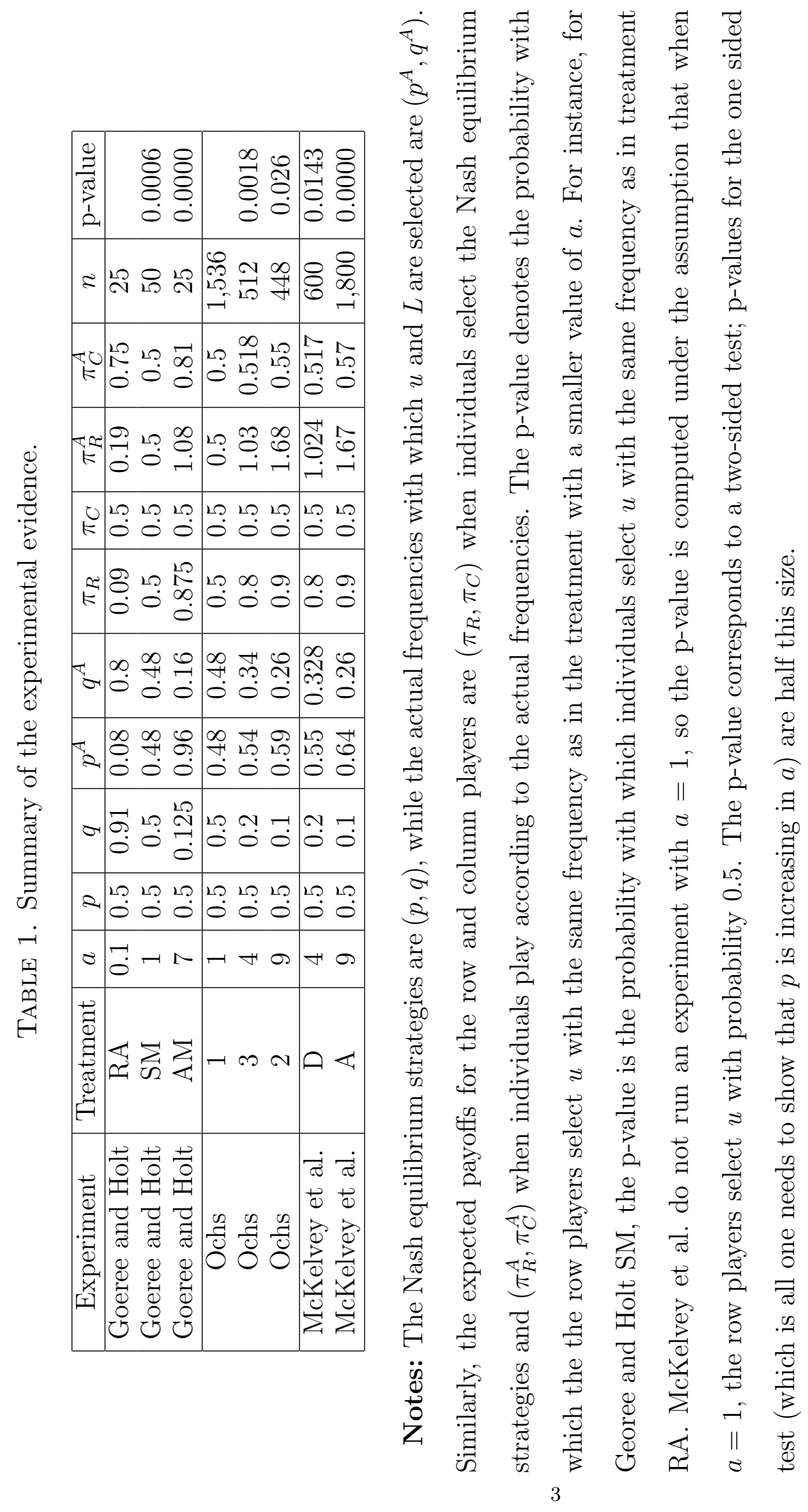


Thus, the experimental evidence strongly points to individuals cooperating. These results may not appear that surprising given the extensive evidence of altruistic behavior observed in games (Fehr and Schmidt [2] and Charness and Rabin [1]). For this reason, these authors have developed game-theoretic equilibrium concepts that account for altruism ([8], [2], [1]). What we find surprising is that these models do not predict cooperative behavior in the generalized game of matching pennies, and thus are inconsistent with our stylized facts.

We consider first a model of boundedly rational players - the quantal response equilibrium - whose comparative statics is consistent with the experimental results. It is already known that the quantal response equilibrium 'fits' experimental data better than the Nash equilibrium [5]. However, it has not been pointed out before that, for the generalized game of matching pennies, the quantal response equilibrium predicts that individuals act cooperatively.

\section{The quANTAL RESPONSE EQUILIBRIUM}

Consider a two-player game in normal form $\left(S_{1}, S_{2}, \pi_{1}, \pi_{2}\right)$, where for $i=1,2, S_{i}$ is the finite set of actions for player $i$, and $\pi_{i}\left(s_{i}, s_{j}\right)$ is the payoff for player $i$ when she selects action $s_{i} \in S_{i}$ and player $j$ selects action $s_{j} \in S_{j}$. Let $\Delta\left(S_{i}\right)$ denote the set of probability distributions (that is, mixed strategies) of player $i$. For each mixed strategy $\sigma_{i} \in \Delta\left(S_{i}\right)$ and each action $s_{i} \in S_{i}, \sigma_{i}\left(s_{i}\right) \geq 0$ denotes the probability that player $i$ will select the action $s_{i} \in S_{i}$ in that mixed strategy. Naturally, $\sum_{s_{i} \in S_{i}} \sigma_{i}\left(s_{i}\right)=1$. With slight abuse of notation $s_{i}$ also denotes the degenerate mixed strategy for which the action $s_{i}$ is selected with probability one. We set $\pi_{i}\left(\sigma_{i}, \sigma_{j}\right)=\sum_{s_{i} \in S_{i}} \sum_{s_{j} \in S_{j}} \pi\left(s_{i}, s_{j}\right) \sigma_{i}\left(s_{i}\right) \sigma_{j}\left(s_{j}\right)$ to be the expected payoff for player $i$ when choosing her action according to the mixed strategy $\sigma_{i}$ given that player $j$ is selecting her actions according to the mixed strategy $\sigma_{j}$.

McKelvey and Palfrey [5] formulate a theory in which players choose their actions based on relative expected utilities and assume other players do so as well. McKelvey and Palfrey's motivate such behavior as arising from players choosing their strategies with error (embodying a form of 'bounded rationality'). The quantal response equilibrium is then obtained as 
the fixed point of this process. For a logit specification of the error structure they derived the following expressions for the quantal response function and associated equilibrium.

Definition Fix $\lambda \geq 0$. The logistic quantal response function of player $i$ to the mixed strategy $\sigma_{j}$, denoted by $L Q R_{i}\left(\sigma_{j}\right)$, is given by

$$
L Q R_{i}\left(\sigma_{j}\right)\left(s_{i}\right)=\frac{e^{\lambda \pi_{i}\left(s_{i} \mid \sigma_{j}\right)}}{\sum_{\hat{s}_{i} \in S_{i}} e^{\lambda u_{i}\left(\hat{s}_{i} \mid \sigma_{j}\right)}} .
$$

The logit equilibrium is a pair of mixed strategies $\left(\sigma_{1}^{L}, \sigma_{2}^{L}\right)$, such that, $\sigma_{1}^{L}=L Q R_{1}\left(\sigma_{2}^{L}\right)$ and $\sigma_{2}^{L}=L Q R_{2}\left(\sigma_{1}^{L}\right)$

When $\lambda=0$, a player selects each action with equal probability. In the limit as $\lambda \rightarrow \infty$, the probability the player selects the action with the highest payoff tends to one, and the equilibrium converges to Nash. Hence, the parameter $\lambda$ may be interpreted as the degree of rationality of the player, where larger values of $\lambda$ correspond to individuals who are more rational (or more strictly speaking, more likely to select a best response). Of course, higher rationality by all players does not lead to higher payoffs as is shown below.

Proposition 1 below states that the logit equilibrium is consistent with the stylized facts discussed in the Section 2.

Proposition 1. For all $\lambda>0$, in the logit equilibrium, $p$ is increasing in a, while $q$ is decreasing in a. Furthermore, for all $a>0, a \neq 1$ both players obtain higher expected payoffs than in the Nash equilibrium.

A formal proof appears in the appendix, but to provide some intuition let us start with $a=1$, that is, the standard symmetric matching pennies. The logit equilibrium is the same as the Nash with $p=q=1 / 2$, since for each player, given her opponent's behavior, each of the two available actions yields the same expected payoff, namely $1 / 2$. If $a$ were increased and Column's behavior remained unchanged, then the payoff to Row of playing $u$ would be greater than that from playing $d$ and so the logistic quantal response would entail a mixed strategy in which Row plays $u$ with a probability $p>1 / 2$ (but unlike the Nash best response, still less than 1). If Row were playing $u$ with a probability $p>1 / 2$, however, Column's 
expected payoff from selecting $R$ would now be higher than from selecting $L$, and in turn her logistic quantal response would entail a mixed strategy with $q<1 / 2$ (but again unlike a Nash response, still greater than 0). Although this change in Column's mixed strategy reduces the expected payoff of Row playing $u$ versus $d$, the algebra in the proof establishes that $q>1 /(1+a)$ which in turn means that this strategic response of Column does not fully offset the direct effect on Row's payoff from the original increase in a. Furthermore, since in the new logit equilibrium $p>1 / 2$ and $1 /(1+a)<q<1 / 2$, it readily follows that the expected payoffs to Row and Column are greater than what they would receive were they playing Nash. By an analogous argument it can readily be shown that were $a$ to be reduced below one, in the new logit equilibrium we would have $p<1 / 2$ and $1 /(1+a)>q>1 / 2$, with expected payoffs to both higher than their respective Nash equilibrium payoffs.

The direct effect of moving $a$ away from one is to induce Row to select one of his actions with a higher probability than the other. By being 'less random' in his action choice (notice the entropy of his quantal best response has fallen), Row has conferred a benefit on Column. Column 'cooperates' by not fully exploiting this opportunity. That is, her quantal response induces her to place more weight on her now preferred action but unlike a best response, the error structure in her action choice leads Row to expect her to choose the action which benefits Row with sufficiently high probability to have Row's quantal response still involve an unequal weighting in choosing between $u$ and $d$.

In a Nash equilibrium, the parameter $a$ does not affect the likelihood with which the row player selects $a$, and thus the column player's payoffs do not change with $a$. Hence the bounded rationality of the players stemming from the possible errors they may make in choosing an action and their awareness that their opponents may make similar mistakes leads to a logit equilibrium of the asymmetric matching pennies game in which both players choose mixed strategies that are more cooperative than if they were both playing Nash with an attendant outcome in which both players have a higher expected payoff than if they were playing a Nash equilibrium. 


\section{Alternative theories}

4.1. Fairness. Individuals may cooperate if their utility function depend not only on the payoff of the game but also on the kindness and the perceived kindness of others. These ideas are formalized in Rabin [8] and described below. For each $\sigma_{j} \in \Delta\left(S_{j}\right)$, let

$$
\Pi\left(\sigma_{j}\right) \equiv\left\{\left(\pi_{i}\left(\sigma_{i}, \sigma_{j}\right), \pi_{j}\left(\sigma_{j}, \sigma_{i}\right)\right): \sigma_{i} \in \Delta\left(S_{i}\right)\right\},
$$

that is, $\Pi\left(\sigma_{j}\right)$ is the set of pair of payoffs for both players attainable by player $i$ when player $j$ is choosing $\sigma_{j} \in \Delta\left(S_{j}\right)$. Let $\pi_{j}^{h}\left(\sigma_{j}\right)$ (respectively, $\pi_{j}^{\ell}\left(\sigma_{j}\right)$ ) denote player $j$ 's highest (respectively, lowest) payoff among points that are Pareto efficient in $\Pi\left(\sigma_{j}\right)$, and let the equitable payoff be

$$
\pi_{j}^{e}\left(\sigma_{j}\right)=\left[\pi_{j}^{h}\left(\sigma_{j}\right)+\pi_{j}^{\ell}\left(\sigma_{j}\right)\right] / 2
$$

Finally, let $\pi_{j}^{\min }\left(\sigma_{j}\right)$ be the worst possible payoff for player $j$ in the set $\Pi\left(\sigma_{j}\right)$.

Definition Suppose player $i$ selects action $s_{i} \in S_{i}$ and believes that player $j$ selects the mixed strategy $\sigma_{j} \in \Delta\left(S_{j}\right)$. Then, Player $i$ 's kindness to player $j$ is

$$
f_{i}\left(s_{i}, \sigma_{j}\right) \equiv \begin{cases}\frac{\pi_{j}\left(\sigma_{j}, s_{i}\right)-\pi_{j}^{e}\left(\sigma_{j}\right)}{\pi_{j}^{h}\left(\sigma_{j}\right)-\pi_{j}^{\min }\left(\sigma_{j}\right)} & \text { if } \pi_{j}^{h}\left(\sigma_{j}\right)>\pi_{j}^{\min }\left(\sigma_{j}\right) \\ 0 & \text { otherwise. }\end{cases}
$$

Definition Player $i$ 's belief about how kind player $j$ is being to him given his belief that player $j$ is playing the mixed strategy $\sigma_{j} \in \Delta\left(S_{j}\right)$ and his belief that player $j$ believes player $i$ is choosing the mixed strategy $\sigma_{i} \in S_{i}$, is given by

$$
\tilde{f}_{j}\left(\sigma_{j}, \sigma_{i}\right)=\frac{\pi_{i}\left(\sigma_{i}, \sigma_{j}\right)-\pi_{i}^{e}\left(\sigma_{i}\right)}{\pi_{i}^{h}\left(\sigma_{i}\right)-\pi_{i}^{\min }\left(\sigma_{i}\right)}
$$

if $\pi_{i}^{h}\left(\sigma_{i}\right)>\pi_{i}^{\min }\left(\sigma_{i}\right)$, and zero otherwise.

Following Rabin [8], in order to incorporate 'fairness' into the payoffs, we assume an individual maximizes a convex combination of his material payoff and his expectation of the product of his belief about how kind player $j$ is being to him and his kindness to player 
$j$ plus one. That is, suppose player $i$ believes that player $j$ is playing the mixed strategy $\sigma_{j} \in \Delta\left(S_{j}\right)$ and furthermore $i$ believes that $j$ believes that $i$ is playing the mixed strategy $\sigma_{i} \in \Delta\left(S_{i}\right)$, then $i$ 's expected payoff from playing the pure strategy $s_{i}$ is given by

$$
U_{i}\left(s_{i}, \sigma_{j}, \sigma_{i}\right)=(1-\alpha) \pi_{i}\left(s_{i}, \sigma_{j}\right)+\alpha \tilde{f}_{j}\left(\sigma_{j}, \sigma_{i}\right)\left[1+f_{i}\left(s_{i}, \sigma_{j}\right)\right]
$$

where $\alpha \in(0,1)$.

Definition $\left(\sigma_{1}^{F}, \sigma_{2}^{F}\right)$ is a fairness equilibrium if for $i=1,2, j \neq i$,

$$
\sigma_{i}^{F}\left(s_{i}\right)>0 \Rightarrow U_{i}\left(s_{i}, \sigma_{j}^{F}, \sigma_{i}^{F}\right) \geq U_{i}\left(s_{i}^{\prime}, \sigma_{j}^{F}, \sigma_{i}^{F}\right) \text { for all } s_{i}^{\prime} \in S_{i}
$$

Proposition 2. The following is a fairness equilibrium of the asymmetric matching pennies game:

$$
\left(\frac{1}{2}, \frac{2-\alpha}{2[(1-\alpha) a+1]}\right)
$$

Furthermore, if $(a-1)[(2+a) \alpha-(1+a)]>0$ then

$$
\left(\frac{1}{2}, \frac{2-3 \alpha}{2[(1-\alpha)(1+a)-\alpha]}\right)
$$

is also a fairness equilibrium.

This result contradicts the experimental evidence presented in Table 1 that shows that $p$ is increasing in $a$. Instead, Row mixes in the fairness equilibrium the same as if the players selected strategies according to the Nash equilibrium. To see why, notice that for Column to play a mixed strategy in equilibrium requires Row's mixed strategy to induce the same payoff to Column from either of her actions $L$ or $R$. That is, $U_{C}(L, p, q)=U_{C}(R, p, q)$, or equivalently,

$$
(1-\alpha)\left[\pi_{C}(L, p)-\pi_{C}(R, p)\right]+\alpha \tilde{f}_{R}(p, q)\left[f_{C}(L, p)-f_{C}(R, p)\right]=0 .
$$

We know from the Nash equilibrium that $\pi_{C}(L, 1 / 2)=\pi_{C}(R, 1 / 2)$. But it is also straightforward to show that $\tilde{f}_{R}(1 / 2, q)=0$ for all $q$, since $\pi_{C}(q, 1 / 2)=1 / 2=\pi_{C}^{e}(q)$. That is, 
if Column believes Row is choosing each of his actions with equal probability, she believes Row is being neither 'kind' nor 'unkind' since such a mixing by Row guarantees Column an expected material payoff equal to the 'equitable' material payoff of $1 / 2$ no matter what Column chooses.

Analogously, we know that for Row to play a mixed strategy in equilibrium requires Column's mixed strategy to induce the same payoff to Row from either of his actions $u$ or $d$. That is, $U_{R}(u, q, p)=U_{R}(d, q, p)$. Or equivalently,

$$
(1-\alpha)\left[\pi_{R}(u, q)-\pi_{C}(d, q)\right]+\alpha \tilde{f}_{C}(q, p)\left[f_{R}(u, q)-f_{R}(d, q)\right]=0 .
$$

Again we know from the Nash equilibrium that $\pi_{R}(u, 1 /(1+a))=\pi_{R}(d, 1 /(1+a))$. But $\tilde{f}_{C}(1 /(1+a), 1 / 2) \neq 0 .{ }^{1}$ Further, in the proof of Proposition 2 we show that

$$
U_{R}\left(u, \frac{1}{1+a}, \frac{1}{2}\right)-U_{R}\left(d, \frac{1}{1+a}, \frac{1}{2}\right)=\alpha\left(\frac{1}{1+a}-\frac{1}{2}\right) .
$$

That is, with $a>1$, at the Nash equilibrium of the asymmetric game $(p=1 / 2, q=1 /(1+a))$, the 'fair-minded' Row player gets a higher payoff from playing $d$ to playing $u$. Column's fairness equilibrium strategy thus entails her playing $L$ with a higher probability than she would if Row had standard preferences in order to make him indifferent between playing $u$ or $d$. But since

$$
\frac{1}{2}>\frac{2-\alpha}{2[(1-\alpha) a+1]}>\frac{1}{1+a},
$$

it immediately follows that both Row and Column enjoy higher expected material payoffs in the fairness equilibrium than they would in the Nash equilibrium. Similarly with $a<1$, Column plays $L$ with a lower probability than she would if Row had standard preferences, and again the expected material payoffs are higher in the fairness equilibrium than in the Nash equilibrium.

4.2. Social preferences. An alternative approach to 'fairness' is to assume a player has a direct regard for other players' material payoff as well as her own. We shall say an individual

${ }^{1}$ Rather, we have $\tilde{f}_{C}(1 / 2, p)=0$, for all $p$, since $\pi_{R}(p, 1 / 2)=(a p+1-p) / 2=\pi_{R}^{e}(p)$. 
has social preferences (see Charness and Rabin [1]) if the utility for individual $i$ when player $i$ selects action $s_{i}$ and player $j$ selects action $s_{j}$ is $U_{i}\left(s_{i}, s_{j}\right)=u_{i}\left(\pi_{i}\left(s_{i}, s_{j}\right), \pi_{j}\left(s_{j}, s_{i}\right)\right)$, where

$$
\begin{gathered}
u_{i}\left(x_{i}, x_{j}\right)=\left(1-\rho r\left(x_{i}, x_{j}\right)-\tau s\left(x_{i}, x_{j}\right)\right) x_{i}+\left(\rho r\left(x_{i}, x_{j}\right)+\tau s\left(x_{i}, x_{j}\right)\right) x_{j}, \\
r\left(x_{i}, x_{j}\right)=\left\{\begin{array}{ll}
1 & \text { if } x_{i}>x_{j} \\
0 & \text { otherwise }
\end{array} \text { and } s\left(x_{i}, x_{j}\right)= \begin{cases}1 & \text { if } x_{i}<x_{j} \\
0 & \text { otherwise. }\end{cases} \right.
\end{gathered}
$$

I.e., we have

$$
\begin{aligned}
u_{i}\left(x_{i}, x_{j}\right) & =x_{i}+r\left(x_{i}, x_{j}\right) \rho\left(x_{j}-x_{i}\right)+s\left(x_{i}, x_{j}\right) \tau\left(x_{j}-x_{i}\right) \\
& =x_{i}-\rho \max \left(0, x_{i}-x_{j}\right)+\tau \max \left(0, x_{j}-x_{i}\right) .
\end{aligned}
$$

Definition A social-welfare equilibrium is a Nash equilibrium of the game with preferences $\left(U_{R}, U_{C}\right)$.

This model was first considered by Fehr and Schmidt [2] with the additional restriction that $\rho \leq-\tau$ and $0 \leq \rho \leq 1$. Charness and Rabin label preferences depending on the parameters values as follows:

- Competitive preferences correspond to $\tau \leq \rho \leq 0$.

- Difference aversion preferences corresponds to $\tau<0<\rho<1$.

- Social-welfare preferences corresponds to $1 \geq \rho \geq \tau>0$

They interpret their experimental evidence as supporting the social-welfare preferences. However, as shown in Proposition 3, none of the preferences considered in [1] are consistent with the experimental evidence in the generalized game of matching pennies.

Proposition 3. The experimental results that for all $a \neq 1$ individuals obtain higher expected payoffs than Nash is inconsistent with the social-welfare equilibrium.

The intuition for this result is the following. With social preferences, the game can be rewritten as described in Figure 2. In order for the game to maintain the mixed strategy equilibrium, the row players prefer outcomes on the main diagonal of the payoff matrix ( $u L$, 


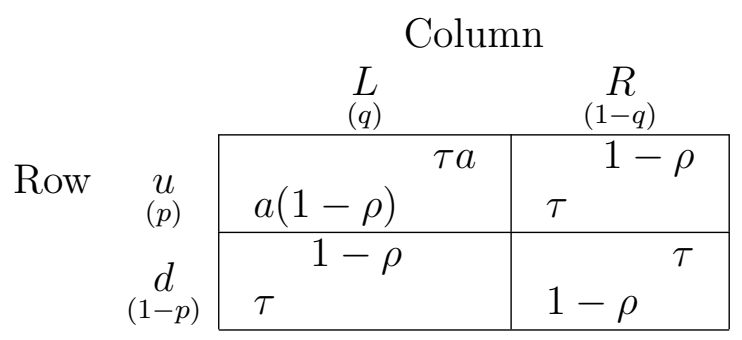

Figure 2. Generalized game of matching pennies, where $a>0$.

$d R)$, while column players prefer outcomes on the off diagonal $(u R, d L)$. This requires that for all $a, \tau a<1-\rho$ and $a(1-\rho)>\tau$. In order for these inequalities to hold for $a$ arbitrarily close to $1, \tau=1-\rho$. However, if $\tau=1-\rho$, the game has no mixed strategy equilibria when $a \neq 1$.

\section{Conclusion}

In this paper we have shown a surprising property of the generalized game of matching pennies. Equilibrium concepts that attempt to model cooperative behavior do not predict that individuals would be able to cooperate in this game. However, a model for how individuals select actions with errors predicts that individuals cooperate. Further, experimental results are consistent with this prediction.

We have defined cooperative behavior as the players choosing mixed strategies that differ from what they would have chosen if they had been playing Nash and leads to both players obtaining payoffs that are higher than Nash. Clearly, the quantal response equilibrium does not have this property for all games. For instance, consider a game that has a unique Nash equilibrium which is Pareto efficient. Then, any equilibrium model in which players do not always select the best response leads to payoff that are lower than Nash. What this suggests, however, is that part of the success of the quantal response equilibrium in analyzing the game of matching pennies may be due to the fact that for this class of games it corresponds to cooperative behavior. 


\section{REFERENCES}

1. Gary Charness and Matthew Rabin, Understanding social preferences with simple tests, Quarterly Journal of Economics 117 (2002), no. 3, 817-869.

2. Ernst Fehr and Klaus M. Schmidt, A theory of fairness, competition and cooperation, Quarterly Journal of Economics 114 (1999), no. 3, 817-868.

3. Jacob K. Goeree and Charles A. Holt, Ten little treasures of game theory and ten intuitive contradictions, American Economic Review 91 (2001), 1402-1422.

4. Jacob K. Goeree, Charles A. Holt, and Thomas R. Palfrey, Risk averse behavior in generalized matching pennies games, Games and Economic Behavior 45 (2003), 97-113.

5. Richard D. McKelvey and Thomas R. Palfrey, Quantal response equilibria for normal form games, Games and Economic Behavior 10 (1995), no. 1, 6-38.

6. Richard D. McKelvey, Thomas R. Palfrey, and Roberto A. Weber, The effects of payoff magnitude and heterogeneity on behavior in $2 \times 2$ games with unique mixed strategy equilibria, Journal of Economic Behavior \& Organization 42 (2000), 523-548.

7. Jack Ochs, Games with unique mixed strategy equilibria: an experimental study, Games and Economic Behavior 10 (1995), 202-217.

8. Matthew Rabin, Incorporating fairness into game theory and economics, American Economic Review $\mathbf{8 3}$ (1993), no. 5, 1281-1302. 


\section{APPENDIX}

Remark In order to describe the experiments in Goeree and Holt as generalized matching pennies games, we subtracted 40 from each player's payoff of 40 and then divided all payoffs by 40. Subtracting a constant from all cells does not change the quantal response, the fairness, or the social welfare equilibrium. Multiplying the payoffs by a constant changes the values of $\lambda$ in the quantal response equilibrium, and the values of $\rho$ and $\tau$ in the socialwelfare equilibrium. This is a reason for why comparisons should be made within different treatments of the same experiment (i.e., Goeree and Holt (AM) and Goeree and Holt (RS)) and not across experiments

Lemma 1. When $a>1$, both players obtain a payoff higher than Nash if and only if $p>1 / 2$ and $q \in\left(\frac{1}{1+a}, 1 / 2\right)$; when $a<1$, both players obtain a payoff higher than Nash if and only if $p<1 / 2$ and $q \in\left(1 / 2, \frac{1}{1+a}\right)$.

Proof. (i) If $q=\frac{1}{a+1}$, the Row's payoffs remain the same. (ii) If $q>\frac{1}{a+1}$, the payoff for action $u$ increases, while the payoff for action $d$ decreases. Therefore, in order for Row's payoffs to increase, requires $p>1 / 2$. The expected payoff for the column player is equal to $p(1-q)+(1-p) q$. Given that $p>1 / 2$, in order for this expression to be greater than one-half, $(1-q)>1 / 2$ or $q<1 / 2$. This is only possible if $a>1$. (iii) If $q<\frac{1}{a+1}$, the payoff for action $u$ decreases, while the payoff for action $d$ increases. Therefore, in order for Row's payoffs to increase, requires $p<1 / 2$. The expected payoff for the column player is equal to $p(1-q)+(1-p) q$. Given that $p<1 / 2$, in order for this expression to be greater than one-half, requires $q>1 / 2$. This is only possible if $a<1$.

Proposition 1. For all $\lambda>0$, in the quantal response equilibrium, $p$ is increasing in $a, q$ is decreasing in a, and both players obtain higher expected payoffs than in the Nash equilibrium. 
Proof. The payoffs are rewritten as $u(0)=0, u(1)=1$, and $u(a)=\alpha$. Let

$$
\begin{aligned}
& f(p, q ; \alpha, \lambda)=\frac{e^{\lambda \alpha q}}{e^{\lambda \alpha q}+e^{\lambda(1-q)}}-p \\
& g(p, q ; \alpha, \lambda)=\frac{e^{\lambda(1-p)}}{e^{\lambda(1-p)}+e^{\lambda p}}-q .
\end{aligned}
$$

Then, a quantal response equilibrium $\left(p^{L}, q^{L}\right)$ satisfies

$$
f\left(p^{L}, q^{L} ; \alpha, \lambda\right)=0, \quad g\left(p^{L}, q^{L} ; \alpha, \lambda\right)=0 .
$$

By the implicit function theorem,

$$
\left(\begin{array}{cc}
f_{p} & f_{q} \\
g_{p} & g_{q} \\
&
\end{array}\right)\left(\begin{array}{c}
p_{\alpha} \\
q_{\alpha}
\end{array}\right)=\left(\begin{array}{c}
-f_{\alpha} \\
-g_{\alpha}
\end{array}\right)
$$

where $f_{p}=g_{q}=-1, g_{\alpha}=0$,

$$
\begin{aligned}
f_{q} & =\frac{\alpha \lambda e^{\lambda \alpha q}\left(e^{\lambda \alpha q}+e^{\lambda(1-q)}\right)+\left(-\lambda \alpha e^{\lambda \alpha q}+\lambda e^{\lambda(1-q)}\right) e^{\lambda \alpha q}}{\left(e^{\lambda \alpha q}+e^{\lambda(1-q)}\right)^{2}} \\
& =\frac{(1+\alpha) \lambda e^{\lambda \alpha q} e^{\lambda(1-q)}}{\left(e^{\lambda \alpha q}+e^{\lambda(1-q)}\right)^{2}}>0 \\
g_{p} & =\frac{-\lambda e^{\lambda(1-p)}\left(e^{\lambda(1-p)}+e^{\lambda p}\right)+\left(\lambda e^{\lambda(1-p)}-\lambda e^{\lambda p}\right) e^{\lambda(1-p)}}{\left(e^{\lambda(1-p)}+e^{\lambda p}\right)^{2}} \\
& =\frac{-2 \lambda e^{\lambda p} e^{\lambda(1-p)}}{\left(e^{\lambda(1-p)}+e^{\lambda p}\right)^{2}}<0 \\
f_{\alpha} & =\frac{\lambda q e^{\lambda \alpha q}\left(e^{\lambda \alpha q}+e^{\lambda(1-q)}\right)-\lambda q e^{\lambda \alpha q} e^{\lambda \alpha q}}{\left(e^{\lambda \alpha q}+e^{\lambda(1-q)}\right)^{2}} \\
& =\frac{\lambda q e^{\lambda(1-q)} e^{\lambda \alpha q}}{\left(e^{\lambda \alpha q}+e^{\lambda(1-q)}\right)^{2}}>0 .
\end{aligned}
$$


By Cramer's rule,

$$
\begin{aligned}
p_{\alpha} & =\frac{f_{\alpha}}{1-f_{q} g_{p}}>0 \\
q_{\alpha} & =\frac{f_{\alpha} g_{p}}{1-f_{q} g_{p}}<0 .
\end{aligned}
$$

Notice that since $f_{q}<0$ and $g_{p}>0,1-f_{q} g_{p}>-f_{q} g_{p}$. Therefore,

$$
q_{\alpha}=\frac{f_{\alpha} g_{p}}{1-f_{q} g_{p}}>\frac{f_{\alpha} g_{p}}{-f_{p} g_{p}}=-\frac{f_{\alpha}}{f_{q}}
$$

It follows that

$$
\begin{aligned}
q_{\alpha} & \geq-\frac{\lambda q e^{\lambda(1-q)} e^{\lambda \alpha q}}{\left(e^{\lambda \alpha q}+e^{\lambda(1-q)}\right)^{2}} \times \frac{\left(e^{\lambda \alpha q}+e^{\lambda(1-q)}\right)^{2}}{(1+\alpha) \lambda e^{\lambda \alpha q} e^{\lambda(1-q)}} \\
& =-\frac{q}{1+\alpha}
\end{aligned}
$$

Notice that when $a=1, \alpha=1, p^{L}(1)=q^{L}(1)=\frac{1}{\alpha+1}=\frac{1}{2}$. Therefore, when $\alpha=1$, $q_{\alpha}>-\frac{q}{1+\alpha}=\frac{1}{(1+\alpha)^{2}}$. Therefore, for all $a>1, q^{L}>\frac{1}{1+\alpha}$. Since $p_{\alpha}>0$ and $q_{\alpha}<0$, it follows that for $a>1, p^{L}>1 / 2$ and $q^{L} \in\left(\frac{1}{1+a}, 1 / 2\right)$. Therefore, by Lemma 1 both players obtain a higher payoff than Nash. The same argument is used for the case when $a<1$.

Proposition 2. The following is a fairness equilibrium of the asymmetric matching pennies game:

$$
\left(\frac{1}{2}, \frac{2-\alpha}{2[(1-\alpha) a+1]}\right)
$$

Furthermore, if $(a-1)[(2+a) \alpha-(1+a)]>0$ then

$$
\left(\frac{1}{2}, \frac{2-3 \alpha}{2[(1-\alpha)(1+a)-\alpha]}\right)
$$

is also a fairness equilibrium. 
Proof. ¿From the payoff matrix in figure 2 we obtain:

\begin{tabular}{l||cccc}
\multicolumn{1}{c||}{$j$} & $\sigma_{j}$ & $\pi_{j}^{h}$ & $\pi_{j}^{\ell}=\pi_{j}^{\min }$ & $\pi_{j}^{e}$ \\
\hline \hline Row & $p$ & $\max (a p, 1-p)$ & $\min (a p, 1-p)$ & $(a p-p+1) / 2$ \\
\hline Column & $q$ & $\max (q, 1-q)$ & $\min (q, 1-q)$ & $1 / 2$
\end{tabular}.

Plugging these values into the definition of $f_{i}(\cdot, \cdot)$ we calculate:

$$
\begin{aligned}
& f_{R}(u, q)=\frac{\pi_{C}(q, u)-\pi_{C}^{e}(q)}{\pi_{C}^{h}(q)-\pi_{C}^{\min }(q)}=\frac{1-q-1 / 2}{|1-2 q|}=\left\{\begin{array}{cl}
1 / 2 & \text { if } q<1 / 2 \\
0 & \text { if } q=1 / 2 \\
-1 / 2 & \text { if } q>1 / 2
\end{array}\right. \\
& f_{R}(d, q)=\frac{\pi_{C}(q, d)-\pi_{C}^{e}(q)}{\pi_{C}^{h}(q)-\pi_{C}^{\min }(q)}=\frac{q-1 / 2}{|1-2 q|}=\left\{\begin{array}{cl}
-1 / 2 & \text { if } q<1 / 2 \\
0 & \text { if } q=1 / 2 \\
1 / 2 & \text { if } q>1 / 2
\end{array}\right. \\
& f_{C}(L, p)=\frac{\pi_{R}(p, L)-\pi_{R}^{e}(p)}{\pi_{R}^{h}(p)-\pi_{R}^{\min }(p)}=\frac{a p-(a p-p+1) / 2}{|a p+p-1|}=\left\{\begin{array}{cl}
-1 / 2 & \text { if } p<\frac{1}{a+1} \\
0 & \text { if } p=\frac{1}{a+1} \\
1 / 2 & \text { if } p>\frac{1}{a+1}
\end{array}\right. \\
& f_{C}(R, p)=\frac{\pi_{R}(p, R)-\pi_{R}^{e}(p)}{\pi_{R}^{h}(p)-\pi_{R}^{\min }(p)}=\frac{(1-p)-(a p-p+1) / 2}{|a p+p-1|}=\left\{\begin{array}{cl}
1 / 2 & \text { if } p<\frac{1}{a+1} \\
0 & \text { if } p=\frac{1}{a+1} \\
-1 / 2 & \text { if } p>\frac{1}{a+1}
\end{array} .\right.
\end{aligned}
$$


And plugging these values into the definition of $\tilde{f}_{i}(\cdot, \cdot)$ yields:

$$
\begin{aligned}
\tilde{f}_{C}(q, p) & =\frac{p a q+(1-p)(1-q)-(a p-p+1) / 2}{|a p+p-1|}=\frac{(p a-1+p)(q-1 / 2)}{|a p+p-1|} \\
& =\left\{\begin{array}{cc}
1 / 2-q & \text { if } p<1 /(1+a) \\
0 & \text { if } p=1 /(1+a) \\
q-1 / 2 & \text { if } p>1 /(1+a)
\end{array}\right. \\
\tilde{f}_{R}(p, q) & =\frac{p(1-q)+(1-p) q-1 / 2}{|1-2 q|}=\frac{(1-2 q)(p-1 / 2)}{|1-2 q|} \\
& =\left\{\begin{array}{cl}
p-1 / 2 & \text { if } q<1 / 2 \\
0 & \text { if } q=1 / 2 \\
1 / 2-p & \text { if } q>1 / 2
\end{array}\right.
\end{aligned}
$$

Thus $(p, q) \in(0,1)^{2}$ is an equilibrium if

$$
\text { Row : } U_{R}(u, q, p)-U_{R}(d, q, p)=0
$$

$$
\text { Column : } U_{C}(L, p, q)-U_{C}(R, p, q)=0
$$

Equation (1) may be reexpressed

$$
(1-\alpha)\left[\pi_{R}(u, q)-\pi_{R}(d, q)\right]+\alpha \tilde{f}_{C}(q, p)\left[f_{R}(u, q)-f_{R}(d, q)\right]=0
$$

and equation (2) becomes

$$
(1-\alpha)\left[\pi_{C}(L, p)-\pi_{C}(R, p)\right]+\alpha \tilde{f}_{R}(p, q)\left[f_{C}(L, p)-f_{C}(R, p)\right]=0
$$

Assuming $p>1 /(1+a)$, then we have by plugging in the appropriate values into (4):

$$
\text { Column : } \begin{array}{ll}
(1-\alpha)(1-2 p)+\alpha(p-1 / 2)=0 & \text { if } q<1 / 2 \\
(1-\alpha)(1-2 p)-\alpha(p-1 / 2)=0 & \text { if } q>1 / 2
\end{array}
$$


But the only solution is $p=1 / 2$. If instead we assume $p<1 /(1+a)$ we have

$$
\text { Column : } \begin{array}{ll}
(1-\alpha)(1-2 p)-\alpha(p-1 / 2)=0 & \text { if } q<1 / 2 \\
(1-\alpha)(1-2 p)+\alpha(p-1 / 2)=0 & \text { if } q>1 / 2
\end{array}
$$

and again, the only solution is $p=1 / 2$.

So fix $p=1 / 2$. Now consider $a>1$. Since $p>1 /(1+a)$ equation (1) becomes

$$
\text { Row : } \begin{array}{ll}
(1-\alpha)(q a-1+q)+\alpha(q-1 / 2)=0 & \text { if } q<1 / 2 \\
(1-\alpha)(q a-1+q)-\alpha(q-1 / 2)=0 & \text { if } q>1 / 2
\end{array}
$$

Which yields for $q<1 / 2$ the solution

$$
q=\frac{1-\alpha+1}{2[(1-\alpha) a+1]}<\frac{1}{2}
$$

And for $q>1 / 2$ the solution is

$$
q=\frac{2-3 \alpha}{2[(1-\alpha)(1+a)-\alpha]}
$$

Now for this value to be greater than $1 / 2$ requires

$$
\begin{array}{r}
2-3 \alpha>(1-\alpha)(1+a)-\alpha>0 \\
\text { or } 3 \alpha-2>a-(1-\alpha)(1+a)>0
\end{array}
$$

But

$$
\begin{aligned}
& 2-3 \alpha>(1-\alpha)(1+a)-\alpha \\
& \Leftrightarrow(a-1) \alpha>(a-1) \Leftrightarrow \alpha>1,
\end{aligned}
$$

a contradiction. Hence

$$
3 \alpha-2>a-(1-\alpha)(1+a) \Leftrightarrow \alpha<1,
$$


and, so when $a>1$, we require

$$
a-(1-\alpha)(1+a)>0 \Leftrightarrow(2+a) \alpha>(1+a) .
$$

It remains to consider the case of $a<1$. Since $p<1 /(1+a)$ equation (1) becomes

$$
\text { Row : } \begin{array}{ll}
(1-\alpha)(q a-1+q)+\alpha(1 / 2-q)=0 & \text { if } q<1 / 2 \\
(1-\alpha)(q a-1+q)-\alpha(1 / 2-q)=0 & \text { if } q>1 / 2
\end{array}
$$

Which yields for $q<1 / 2$ the solution

$$
q=\frac{2-3 \alpha}{2[(1-\alpha)(1+a)-\alpha]}
$$

which is indeed in the open interval $(0,1 / 2)$ if $\alpha<(1+a) /(2+a)$. For $q>1 / 2$ we have

$$
q=\frac{2-\alpha}{2[(1-\alpha) a+1]}>1 / 2
$$

Proposition 3. In a social-welfare equilibrium, for some $a \neq 1 / 2$, one of the players obtains a payoff that is lower or equal to the Nash payoff.

Proof. Consider the case $a>1$. In order for both players to obtain a payoff higher than Nash, $p>1 / 2$ and $q \in\left(\frac{1}{1+a}, 1 / 2\right)$. Thus, there are two possible equilibria: both players mix, and only the column player mixes.

First suppose that in equilibrium all actions are selected with positive probability. In a mixed strategy equilibrium the expected payoff for Row of $u$ is the same as the expected payoff of $d$. This means that

$$
q(a-\rho a)+(1-q) \tau=q \tau+(1-q)(1-\rho),
$$

or

$$
q=\frac{1-\tau-\rho}{1+a-2 \tau-\rho-a \rho}=\frac{(1-\tau-\rho)}{(1-\tau-\rho)+(a-\tau-a \rho)} .
$$


Similarly, the expected payoff of Column for $L$ is the same as the expected payoff for $R$ :

$$
p(\tau a)+(1-p)(1-\rho)=p(1-\rho)+(1-p) \tau
$$

or

$$
p=\frac{1-\tau-\rho}{2-\tau-a \tau-2 \rho}=\frac{1-\tau-\rho}{(1-\tau-\rho)+(1-a \tau-\rho)}
$$

In order for $p>1 / 2, \tau>\frac{1-\rho}{a}$. In order for $q<1 / 2, a(1-\rho)>\tau$. In order for both of these inequalities to hold for all $a, \tau=1-\rho$. This implies that $q=0$ which contradicts our assumption that all actions are played with positive probability.

Second, suppose the row player selects $u$ and column player mixes. Then, the expected payoff of Column for $L$ is the same as the expected payoff for $R$ :

$$
\tau a=1-\rho,
$$

which can hold for at most one value of $a$.

Rice UnIVERsity

E-mail address: rboylan@rice.edu 